\title{
Weight Least Squares Algorithm for Rational Models with Outliers
}

\author{
Lixin Lv $\mathbb{D}$ and Donglei Lu \\ Wuxi Vocational College of Science and Technology, Wuxi 214028, China \\ Correspondence should be addressed to Lixin Lv; 1201053@wxsc.edu.cn \\ Received 29 January 2020; Accepted 23 March 2020; Published 14 April 2020 \\ Academic Editor: Quanmin Zhu \\ Copyright (c) 2020 Lixin Lv and Donglei Lu. This is an open access article distributed under the Creative Commons Attribution \\ License, which permits unrestricted use, distribution, and reproduction in any medium, provided the original work is \\ properly cited. \\ A weight least squares algorithm is developed for rational models with outliers in this paper. Different weights are assigned for
each cost function, and by calculating the derivatives of these cost functions, the parameter estimates can be estimated. Compared
with the traditional least squares algorithm, the proposed algorithm can remove the bad effect caused by the outliers, thus has
more accurate parameter estimates. A simulation example is proposed to validate the effectiveness of the proposed algorithm.
}

\section{Introduction}

System identification can be roughly divided into two kinds: the structure identification and the parameter estimation [1-4]. Compared to the parameter estimation, structure identification is more difficult, because one should choose the available products from numerous product pool. Once the structure is determined, parameter estimation is involved [5-7]. Recently, a lot of parameter estimation algorithms are proposed, including the least squares (LS) algorithm [8, 9], the gradient algorithm [10, 11], the particle swarm optimization algorithm, and the expectation maximization algorithm $[12,13]$. Among these algorithms, the LS algorithm is to find a vector that is a local minimizer to a function that is a sum of squares; thus, it is the simplest algorithm and is widely used.

Although the LS algorithm is applied to many different kinds of nonlinear models, e.g., bilinear models $[14,15]$, polynomial nonlinear models $[16,17]$, hard nonlinear models, and rational nonlinear models [18, 19], it also brings a challenging problem: heavy computational effort caused by the inverse matrix calculation. In order to overcome this difficulty, some online algorithms are proposed. For example, Liu and Ding proposed an auxiliary model-based recursive generalized least squares algorithm for multivariate outputerror autoregressive system [20], Zhu provided an implicit least squares algorithm for nonlinear rational models [21], Wang et al. developed a recursive LS (RLS) algorithm for
Hammerstein models [22]. However, all the systems in the above literature have normal data, when some data of the systems are abnormal, those algorithms may be invalid.

Processes in industry usually suffer from outliers in measurement data [23-25], which make the identification of the process a challenging problem. Thus, a reliable estimation of the systems with outliers is essential to efficient process identification $[26,27]$. Zhao et al. provided a VB approach for ARX models with outliers and assumed that all the variables are available and the precise interval of timedelays is known a priori [28]. Jin et al. proposed a conventional approach to nonlinear process models with outliers [29]. Both these two algorithms are off-line algorithms, which update the parameters through all the collected data, and thus have heavy computational efforts especially for large-scale systems.

In this paper, a weight LS algorithm is proposed for rational models with outliers. By introducing different weights for each cost function, the cost functions of the outliers can be neglected. Furthermore, to reduce the computational efforts, a weight recursive least squares (WRLS) is also derived. Both these two algorithms can yield the optimal parameter estimates. Briefly, the paper is listed as follows. Section 2 introduces the rational model and the traditional LS algorithm. Section 3 develops the weight LS algorithm and the weight RLS algorithm. Section 4 provides a simulation example. Finally, Section 5 gives a conclusion of this paper. 


\section{Rational Model with Outliers}

The rational model with outliers is written by

$$
\begin{aligned}
& y_{1}(t)=\frac{\alpha(t)+e_{1}(t)}{\beta(t)}, \\
& y_{2}(t)=\frac{\alpha(t)+e_{2}(t)}{\beta(t)},
\end{aligned}
$$

where $y_{1}(t)$ is the normal output, $y_{2}(t)$ is the abnormal output, $e_{1}(t)$ a normal stochastic white noise with zero mean and variance $\sigma^{2}, e_{2}(t)$ the outliers of the rational model, whose noise to signal ratio is larger, and $\alpha(t)$ and $\beta(t)$ are the structures of the rational model that can be expressed as

$$
\begin{aligned}
& \alpha(t)=\varphi^{\mathrm{T}}(t) \theta_{a}, \\
& \beta(t)=\psi^{\mathrm{T}}(t) \theta_{b} .
\end{aligned}
$$

The information vectors $\varphi(t)=\left[\varphi_{1}(t), \ldots, \varphi_{n}(t)\right]^{\mathrm{T}}$ and $\psi(t)=\left[\psi_{1}(t), \ldots, \psi_{m}(t)\right]^{\mathrm{T}}$ are the products of past inputs $\{u(t-1), u(t-2), \ldots\}$ and past outputs $\{y(t-1), y(t-2)$, $\ldots\}$, and the structures of $\varphi(t)$ and $\psi(t)$ are known in prior, $\theta_{a}$ and $\theta_{b}$ are the unknown parameters to be estimated and can be expressed as

$$
\begin{aligned}
& \theta_{a}=\left[a_{1}, a_{2}, \ldots, a_{n}\right]^{\mathrm{T}}, \\
& \theta_{b}=\left[b_{1}, b_{2}, \ldots, b_{m}\right]^{\mathrm{T}} .
\end{aligned}
$$

In application, the number of the abnormal data is far smaller than that of the normal data. Assume that we have collected $L$ input-output data, the number of the normal data is $L_{1}$ and the abnormal data is $L_{2}, L_{1}+L_{2}=L, L_{1} \gg L_{2}$. Without loss of generality, assume that $b_{1}=1$, then the rational model is transformed into

$$
\begin{aligned}
\bar{y}(t) & =\phi(t) v+e_{i}(t), \quad i=1,2, \\
\bar{y}(t) & =y(t) \psi_{1}(t), \\
\phi(t) & =\left[\varphi_{1}(t), \ldots, \varphi_{n}(t),-y(t) \psi_{2}(t), \ldots,-y(t) \psi_{m}(t)\right]^{\mathrm{T}}, \\
v & =\left[a_{1}, \ldots, a_{n}, b_{2}, \ldots, b_{m}\right]^{\mathrm{T}} .
\end{aligned}
$$

Collect $L$ input and output data and define

$$
\begin{aligned}
& \bar{Y}(L)=[\bar{y}(L), \bar{y}(L-1), \ldots, \bar{y}(1)]^{\mathrm{T}} \in \mathbb{R}^{L}, \\
& \Phi(L)=[\phi(L), \ldots, \phi(1)]^{\mathrm{T}} \in \mathbb{R}^{L \times(m+n-1)}, \\
& E(L)=\left[e_{i}(L), \ldots, e_{i}(1)\right]^{\mathrm{T}} \in \mathbb{R}^{L},
\end{aligned}
$$

where $i=1$ or 2 . We have

$$
\bar{Y}(L)=\Phi(L) v+E(L) .
$$

Define the cost function

$$
J(v)=\frac{1}{2}[\bar{Y}(L)-\Phi(L) v]^{2},
$$

then the following LS algorithm can be obtained

$$
\widehat{v}=\left[\Phi^{\mathrm{T}}(L) \Phi(L)\right]^{-1} \Phi^{\mathrm{T}}(L) \bar{Y}(L) .
$$

Remark 1. In the LS algorithm, all the data are used to update the parameter estimates; thus, the outliers cause extremely bad influence to the estimates.

\section{Weight Based Least Squares Algorithm}

In order to get more accurate parameter estimates, one should weak/eliminate the bad influence caused by the outliers. Therefore, a weight least squares algorithm is proposed in this section.

3.1. Weight LS Algorithm. Rewrite the cost function as

$$
J(v)=\frac{1}{2}[\bar{Y}(L)-\Phi(L) v]^{2},
$$

which can be transformed into

$$
J(v)=\frac{1}{2} \sum_{t=1}^{L}[\bar{y}(t)-\phi(t) v]^{2}
$$

Clearly, each cost function plays the same role in estimating the parameters when using the traditional LS algorithm. For this reason, we can introduce different weights for each cost function, e.g., small weight for the cost function of the outliers and large weight for the cost function of the normal data.

It follows that the new cost function can be written by

$$
J(v)=\frac{1}{2} \sum_{t=1}^{L} \lambda^{L-t}[\bar{y}(t)-\phi(t) v]^{2}, \quad 0<\lambda<1 .
$$

Taking the derivative of $J(v)$ with respect to $v$ and then equating it to zero give

$$
\widehat{v}=\left[\sum_{t=1}^{L} \lambda^{L-t} \phi(t) \phi^{\mathrm{T}}(t)\right]^{-1}\left[\sum_{t=1}^{L} \lambda^{L-t} \phi(t) \bar{y}(t)\right] .
$$

Remark 2. Based on equations (12) and (13), we can get that each cost function has different weights, and the latest data have larger weights.

Taking the conditional expectation on both sides of (13) yields

$$
\begin{aligned}
E[\widehat{v}] & =E\left[\sum_{t=1}^{L} \lambda^{L-t} \phi(t) \phi^{\mathrm{T}}(t)\right]^{-1}\left[\sum_{t=1}^{L} \lambda^{L-t} \phi(t) \bar{y}(t)\right] \\
& =E\left[\sum_{t=1}^{L} \lambda^{L-t} \phi(t) \phi^{\mathrm{T}}(t)\right]^{-1}\left[\sum_{t=1}^{L} \lambda^{L-t} \phi(t)(\phi(t) v+e(t))\right] \\
& =v+\left[\sum_{t=1}^{L} \lambda^{L-t} \phi(t) \phi^{\mathrm{T}}(t)\right]^{-1} E\left[\sum_{t=1}^{L} \lambda^{L-t} \phi(t) e(t)\right] .
\end{aligned}
$$


Since $e(t)$ is independent of $\phi(t)$, which means that

$$
\left[\sum_{t=1}^{L} \lambda^{L-t} \phi(t) \phi^{\mathrm{T}}(t)\right]^{-1} E\left[\sum_{t=1}^{L} \lambda^{L-t} \phi(t) e(t)\right]=0
$$

Therefore, we have

$$
E[\widehat{v}]=v .
$$

Unfortunately, the W-LS algorithm needs to perform the inverse matrix calculation $\left[\sum_{t=1}^{L} \lambda^{L-t} \phi(t) \phi^{\mathrm{T}}(t)\right]^{-1}$, if the order $m+n-1$ is large, and the computational effort is heavy. To overcome this difficulty, we derive a weight recursive least squares algorithm in the following subsection.

3.2. Weight Recursive Least Squares Algorithm. Define

$$
\begin{aligned}
P^{-1}(L)= & \sum_{t=1}^{L} \lambda^{L-t} \phi(t) \phi^{\mathrm{T}}(t)=\lambda^{L-1} \phi(1) \phi^{\mathrm{T}}(1)+\lambda^{L-2} \phi(2) \\
& \cdot \phi^{\mathrm{T}}(2)+\cdots+\lambda \phi(L-1) \phi^{\mathrm{T}}(L-1)+\phi(L) \phi^{\mathrm{T}}(L), \\
P^{-1}(L-1)= & \sum_{t=1}^{L-1} \lambda^{L-1-t} \phi(t) \phi^{\mathrm{T}}(t)=\lambda^{L-2} \phi(1) \phi^{\mathrm{T}}(1) \\
& +\lambda^{L-3} \phi(2) \phi^{\mathrm{T}}(2)+\cdots+\phi(L-1) \phi^{\mathrm{T}}(L-1) .
\end{aligned}
$$

The above two equations give rise to

$$
P^{-1}(L)=\lambda P^{-1}(L-1)+\phi(L) \phi^{\mathrm{T}}(L) .
$$

Lemma 1. [30]. Assume that $A \in \mathbb{R}^{n \times n}, B \in \mathbb{R}^{n \times m}$, and $C \in \mathbb{R}^{m \times n}$, then the following equality holds:

$$
[A+B C]^{-1}=A^{-1}-A^{-1} B\left(I+C A^{-1} B\right)^{-1} C A^{-1} \text {. }
$$

According to Lemma 1, $P(L)$ can be written by

$$
\begin{aligned}
P(L) & =\left[P^{-1}(L)\right]^{-1}=\left[\lambda P^{-1}(L-1)+\phi(L) \phi^{\mathrm{T}}(L)\right]^{-1} \\
& =\frac{1}{\lambda} P(L-1)-\frac{\left(1 / \lambda^{2}\right) P(L-1) \phi(L) \phi^{\mathrm{T}}(L) P(L-1)}{1+(1 / \lambda) \phi^{\mathrm{T}}(L) P(L-1) \phi(L)} \\
& =\frac{1}{\lambda} P(L-1)-\frac{P(L-1) \phi(L) \phi^{\mathrm{T}}(L) P(L-1)}{\lambda^{2}+\lambda \phi^{\mathrm{T}}(L) P(L-1) \phi(L)} .
\end{aligned}
$$

The parameter estimates by using the W-LS algorithm are rewritten by

$$
\begin{aligned}
\widehat{v}(L) & =\left[\sum_{t=1}^{L} \lambda^{L-t} \phi(t) \phi^{\mathrm{T}}(t)\right]^{-1}\left[\sum_{t=1}^{L} \lambda^{L-t} \phi(t) \bar{y}(t)\right] \\
& =P(L)\left[\sum_{t=1}^{L} \lambda^{L-t} \phi(t) \bar{y}(t)\right]=P(L)\left[\sum_{t=1}^{L-1} \lambda^{L-t} \phi(t) \bar{y}(t)+\phi(L) \bar{y}(L)\right] \\
& =P(L)\left[\lambda \sum_{t=1}^{L-1} \lambda^{L-1-t} \phi(t) \bar{y}(t)+\phi(L) \bar{y}(L)\right] \\
& =P(L)\left[\lambda\left[\sum_{t=1}^{L-1} \lambda^{L-1-t} \phi(t) \phi^{\mathrm{T}}(t)\right]\left[\sum_{t=1}^{L-1} \lambda^{L-1-t} \phi(t) \phi^{\mathrm{T}}(t)\right]^{-1} \sum_{t=1}^{L-1} \lambda^{L-1-t} \phi(t) \bar{y}(t)+\phi(L) \bar{y}(L)\right] \\
& =P(L)\left[\lambda\left[\sum_{t=1}^{L-1} \lambda^{L-1-t} \phi(t) \phi^{\mathrm{T}}(t)\right] \widehat{v}(L-1)+\phi(L) \bar{y}(L)\right] \\
& =P(L)\left[\lambda P^{-1}(L-1) \widehat{v}(L-1)+\phi(L) \bar{y}(L)\right] .
\end{aligned}
$$

According to equation (18), we have

$$
\lambda P^{-1}(L-1)=P^{-1}(L)-\phi(L) \phi^{\mathrm{T}}(L) .
$$

Therefore, equation (21) is simplified to

$$
\begin{aligned}
\widehat{v}(L) & =P(L)\left[\left[P^{-1}(L)-\phi(L) \phi^{\mathrm{T}}(L)\right] \widehat{v}(L-1)+\phi(L) \bar{y}(L)\right] \\
& =\widehat{v}(L-1)+P(L) \phi(L)\left[\bar{y}(L)-\phi^{\mathrm{T}}(L) \widehat{v}(L-1)\right] .
\end{aligned}
$$

The parameter estimates $\widehat{v}(t)$ by using the weight recursive least squares (W-RLS) algorithm are listed as follows:

$$
\begin{aligned}
& \widehat{v}(t)=\widehat{v}(t-1)+P(t) \phi(t)\left[\bar{y}(t)-\phi^{\mathrm{T}}(t) \widehat{v}(t-1)\right] \\
& \phi(t)=\left[-y(t) \psi_{2}(t), \ldots,-y(t) \psi_{m}(t), \varphi_{1}(t), \ldots, \varphi_{n}(t)\right]^{\mathrm{T}}
\end{aligned}
$$

$$
\bar{y}(t)=y(t) \psi_{1}(t)
$$

$$
P(t)=\frac{1}{\lambda} P(t-1)-\frac{P(t-1) \phi(t) \phi^{\mathrm{T}}(t) P(t-1)}{\lambda^{2}+\lambda \phi^{\mathrm{T}}(t) P(t-1) \phi(t)}, \quad 0<\lambda<1 .
$$


The W-RLS algorithm consists of the following iterations:

(1) Let $u(t)=0, y(t)=0, t \leq 0$, and give a small positive number $\varepsilon$ and a positive number $0<\lambda<1$.

(2) Let $t=1 \widehat{v}(0)=\mathbf{1} / p_{0}$ with $\mathbf{1}$ being a column vector whose entries are all unity and $p_{0}=10^{6}, P(0)=p_{0} I$.

(3) Collect $y(t)$ and $u(t)$.

(4) Form $\bar{y}(t)$ by equation (26).

(5) Form $\phi(t)$ according to equation (25).

(6) Compute $P(t)$ by equation (27).

(7) Update the parameter estimation vector $\widehat{v}(t)$ by equation (24).

(8) Compare $\widehat{v}(t) \quad$ and $\quad \widehat{v}(t-1)$ : if $(\|\widehat{v}(t)-\widehat{v}(t-1)\| /\|\widehat{v}(t)\|) \leqslant \mathcal{E}$, then terminate the procedure and obtain $\widehat{v}(t)$; otherwise, increase $t$ by 1 and go to step 3 .

\subsection{Property of the $W$-RLS Algorithm}

Lemma 2. Assume that $A \in \mathbb{R}^{n \times n}$ and $B \in \mathbb{R}^{n \times n}$ are both symmetric positive semidefinite matrices, then $A+B$ is also a symmetric positive semidefinite matrix.

Proof. Since $A$ and $B$ are symmetric positive semidefinite matrices, for a random nonzero vector $X \in \mathbb{R}^{n}$, the following inequalities hold:

$$
\begin{aligned}
& X^{\mathrm{T}} A X \geq 0, \\
& X^{\mathrm{T}} B X \geq 0 .
\end{aligned}
$$

It gives rise to

$$
X^{\mathrm{T}}(A+B) X \geq 0
$$

which means that $A+B$ is also a symmetric positive semidefinite matrix.

Lemma 3. Assume that $A \in \mathbb{R}^{n \times n}$ and $B \in \mathbb{R}^{n \times n}$ are both positive definite symmetric matrices. The largest eigenvalue of the matrix $A$ is $a$, and the largest eigenvalue of the matrix $B$ is $b$. Then the largest eigenvalue of the matrix $A+B$ is $a+b$.

Proof. Since $A$ and $B$ are positive definite symmetric matrices, and the largest eigenvalues are $a$ and $b$, respectively. Then we have

$$
\begin{gathered}
a I-A \geq 0, \\
b I-B \geq 0 .
\end{gathered}
$$

Clearly, $a I-A$ and $b I-B$ are symmetric positive semidefinite matrices. We have

$$
(a+b) I-(A+B) \geq 0 .
$$

Therefore, the largest eigenvalue of the matrix $A+B$ is $a+b$.
Theorem 2. For the rational model in (2), the parameter estimate $\widehat{v}(t)$ by using the $W$-RLS algorithm is expressed by equations (24)-(27). Then we have

$$
\lim _{t \rightarrow \infty} \widehat{v}(t)=v \text {. }
$$

Proof. Rewrite $\widehat{v}(t)$ as follows:

$$
\widehat{v}(t)=\widehat{v}(t-1)+P(t) \phi(t)\left[\bar{y}(t)-\phi^{\mathrm{T}}(t) \widehat{v}(t-1)\right],
$$

and the following equation can be obtained

$$
\begin{aligned}
\widehat{v}(t)-v= & \widehat{v}(t-1)-v+P(t) \phi(t)\left[\phi^{\mathrm{T}}(t) v+v(t)\right. \\
& \left.-\phi^{\mathrm{T}}(t) \widehat{v}(t-1)\right] .
\end{aligned}
$$

Taking the conditional expectation on both sides of (34) gets

$$
\begin{aligned}
E[\widehat{v}(t)-v]= & E\left[\left[I-P(t) \phi(t) \phi^{\mathrm{T}}(t)\right][\widehat{v}(t)-v]\right] \\
& +E[P(t) \phi(t) e(t)] .
\end{aligned}
$$

$e(t)$ is Gaussian noise and satisfies

$$
E(e(t))=0
$$

It follows that

$$
E[\widehat{v}(t)-v]=E\left[\left[I-P(t) \phi(t) \phi^{\mathrm{T}}(t)\right][\widehat{v}(t)-v]\right] .
$$

Since

$$
\begin{aligned}
P(t) & =\left[P^{-1}(t)\right]^{-1}=\left[\sum_{i=1}^{t} \lambda^{t-i} \phi(i) \phi^{\mathrm{T}}(i)\right]^{-1} \\
& =\left[\sum_{i=1}^{t-1} \lambda^{t-i} \phi(i) \phi^{\mathrm{T}}(i)+\phi(t) \phi^{\mathrm{T}}(t)\right]^{-1} .
\end{aligned}
$$

According to Lemmas 2 and 3 we have

$$
\left\|\left[I-P(t) \phi(t) \phi^{\mathrm{T}}(t)\right]\right\|<1 .
$$

Assume that the largest eigenvalue of the matrix $I-$ $P(t) \phi(t) \phi^{\mathrm{T}}(t)$ is $\lambda(t)$, and let

$$
\lambda_{\max }=\{\lambda(1), \lambda(2), \ldots,\} .
$$

Clearly, $0<\lambda_{\max }<1$.

It follows that

$$
\lim _{t \longrightarrow \infty}\|\widehat{v}(t)-v\|=\lim _{t \longrightarrow \infty} \lambda_{\max }^{t}\|\widehat{v}(0)-v\|=0 .
$$

Then the parameter estimate by using the W-RLS algorithm is asymptotic convergent.

Remark 3. Unlike the W-LS algorithm, the W-RLS algorithm avoids the inverse matrix calculation, thus has less computational efforts.

Remark 4. At the sampling instant $t$, the W-RLS algorithm uses the data up to and including time $t$, while the stochastic 


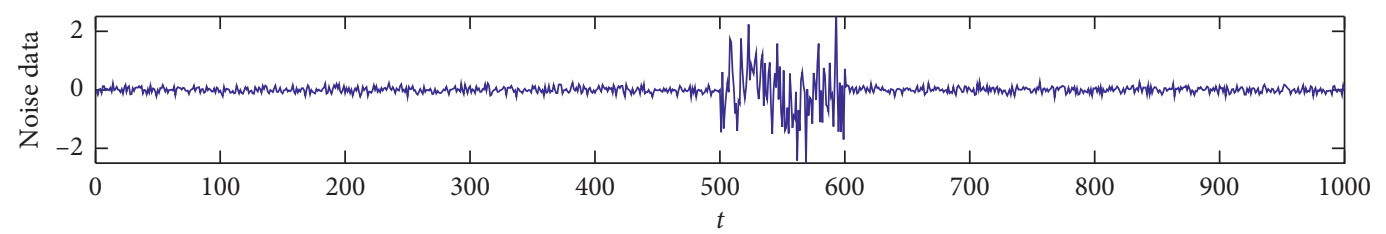

(a)

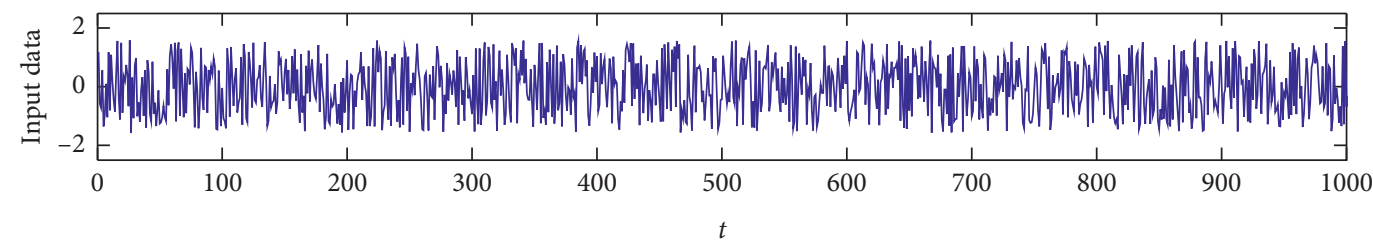

(b)

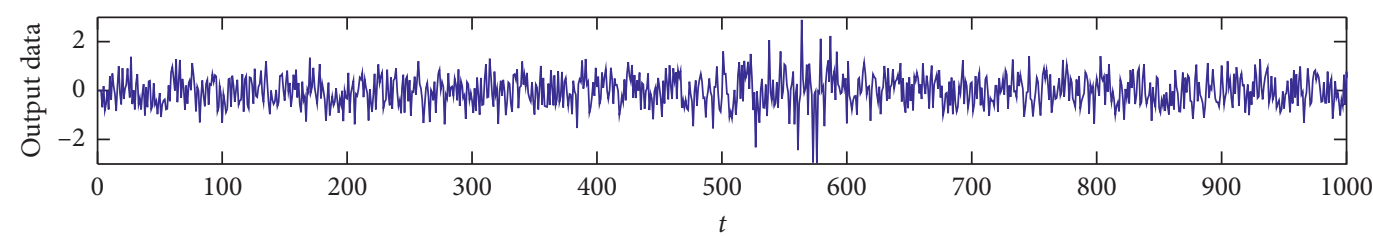

(c)

Figure 1: The simulation data.

TABLE 1: The parameter estimates and errors.

\begin{tabular}{lcccccc}
\hline Algorithms & $a_{1}$ & $a_{2}$ & $a_{3}$ & $b_{2}$ & $b_{3}$ & $\tau(\%)$ \\
\hline LS & 0.15181 & 0.07996 & 0.79217 & 0.51845 & 0.51375 & 41.06137 \\
W-LS & 0.12532 & 0.12418 & 1.00963 & 0.98697 & 0.98437 & 4.67610 \\
\hline True values & 0.20000 & 0.10000 & 1.00000 & 1.00000 & 1.00000 \\
\hline
\end{tabular}

TABLE 2: The parameter estimates and errors.

\begin{tabular}{|c|c|c|c|c|c|c|c|}
\hline Algorithms & $t$ & $a_{1}$ & $a_{2}$ & $a_{3}$ & $b_{2}$ & $b_{3}$ & $\tau(\%)$ \\
\hline \multirow{7}{*}{ RLS } & 100 & 0.19112 & 0.12398 & 0.95403 & 0.89402 & 0.84710 & 11.07001 \\
\hline & 300 & 0.18911 & 0.11181 & 0.95851 & 0.89135 & 0.91121 & 8.42857 \\
\hline & 600 & 0.02324 & -0.01496 & 0.46701 & -0.37791 & -0.29143 & 113.00607 \\
\hline & 900 & 0.04782 & 0.00113 & 0.49319 & -0.31770 & -0.24903 & 108.43468 \\
\hline & 1200 & 0.05971 & 0.01433 & 0.51901 & -0.25419 & -0.21230 & 104.03382 \\
\hline & 1500 & 0.07107 & 0.02377 & 0.54218 & -0.19289 & -0.17885 & 99.91320 \\
\hline & 1800 & 0.08303 & 0.02983 & 0.57281 & -0.12658 & -0.12806 & 94.83039 \\
\hline \multirow{8}{*}{ W-RLS } & 100 & 0.19100 & 0.12362 & 0.94507 & 0.88143 & 0.82101 & 12.77180 \\
\hline & 300 & 0.18310 & 0.09174 & 0.95825 & 0.88932 & 0.92104 & 8.21469 \\
\hline & 600 & -0.07442 & -0.12394 & 0.34539 & -0.54102 & -0.30873 & 123.36106 \\
\hline & 900 & 0.10473 & 0.04088 & 0.61333 & -0.03655 & 0.05515 & 83.55360 \\
\hline & 1200 & 0.17230 & 0.08650 & 0.91166 & 0.78224 & 0.75087 & 19.68922 \\
\hline & 1500 & 0.17447 & 0.09915 & 0.92978 & 0.83961 & 0.77437 & 16.41848 \\
\hline & 1800 & 0.18480 & 0.09849 & 0.97901 & 0.95148 & 0.93699 & 4.79017 \\
\hline & True values & 0.20000 & 0.10000 & 1.00000 & 1.00000 & 1.00000 & \\
\hline
\end{tabular}

gradient algorithm only uses the current data $y(t), u(t)$; thus, the W-RLS algorithm has a quicker convergence rate than that of the stochastic gradient algorithm.

\section{Example}

Consider the rational model proposed in [31], 


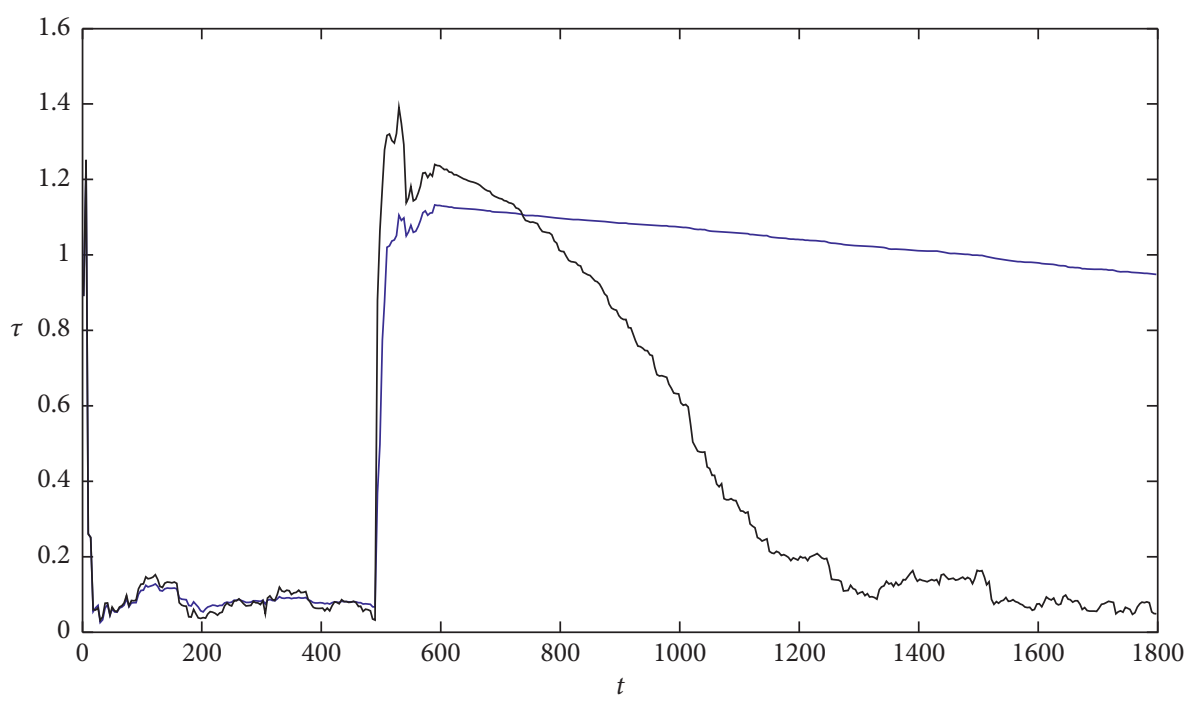

- RLS

Figure 2: The parameter estimation errors $\tau$ versus.t.

$y(t)=\frac{0.2 y(t-1)+0.1 y(t-1) u(t-1)+u(t-1)+e(t)}{1+y^{2}(t-1)+y^{2}(t-2)}$

Then, one can get

$$
\begin{aligned}
y(t)= & 0.2 y(t-1)+0.1 y(t-1) u(t-1) \\
& +u(t-1)-y(t) y^{2}(t-1)-y(t) y^{2}(t-2)+e(t)
\end{aligned}
$$

where $\{u(t)\}$ is a input sequence with zero mean and unit variance $\{e(t)\}$ is a white noise with zero mean and variance $\sigma^{2}$. In simulation, we collect 1800 input-output data, and the data from 500-600 are outliers. The noise to signal ratio is $260.2 \%$ when $t \in[500,600]$, and $2.78 \%$ when $t \in[0,499] \cup[601,1800]$. The simulation data are shown in Figure $1, t \in[1,1000]$, it shows the noise and output data from 500 to 600 are outliers.

Apply the LS and W-LS algorithms to the rational model $(\lambda=0.9)$. The parameter estimates and their estimation errors $\tau:=(\|\widehat{v}-v\| /\|v\|)$ are shown in Table 1 .

Next, use the RLS and the W-RLS algorithms for this rational models $(\lambda=0.9)$, the parameter estimates and their estimation errors $\tau:=(\|\widehat{v}-v\| /\|v\|)$ are shown in Table 2 and Figure 2.

Then the following findings can be obtained: (1) Table 1 shows that the W-LS algorithm is more effective than the LS algorithm; (2) Table 2 and Figure 2 demonstrate that the parameter estimates by using the W-RLS algorithm are more accurate than those by using the RLS algorithm.

\section{Conclusions}

This paper proposes a weight least squares algorithm for rational models with outliers. By assigning the weights for each data set, the proposed algorithm can obtain more accurate parameter estimates when compared with the traditional least squares algorithm. Furthermore, a weight recursive least squares algorithm is derived to decrease the computational efforts. The convergence analysis and simulation example show that the proposed algorithm is effective.

\section{Data Availability}

There are no data in our manuscript.

\section{Conflicts of Interest}

The authors declare that they have no conflicts of interest.

\section{Acknowledgments}

This study was funded by the Natural Science Foundation for Colleges and Universities in Jiangsu Province (No. 18KJB120009).

\section{References}

[1] T. Söderström and U. Soverini, "Errors-in-variables identification using maximum likelihood estimation in the frequency domain," Automatica, vol. 79, pp. 131-143, 2017.

[2] D. Wang, S. Zhang, M. Gan, and J. Qiu, "A novel EM identification method for Hammerstein systems with missing output data," IEEE Transactions on Industrial Informatics, vol. 16, no. 4, pp. 2500-2508, 2020.

[3] F. Giri, Y. Rochdi, and F. Z. Chaoui, "Hammerstein systems identification in presence of hard nonlinearities of preload and dead-zone type," IEEE Transactions on Automatic Control, vol. 54, no. 9, pp. 2174-2178, 2009.

[4] J. Na, Z. Yang, S. Kamal, L. Hu, W. Wang, and Y. Zhou, "Bioinspired learning and adaptation for optimization and control 
of complex systems," Complexity, vol. 2019, Article ID 9325364, 3 pages, 2019.

[5] G. Y. Chen, M. Gan, C. L. P. Chen, and H. X. Li, "A regularized variable projection algorithm for separable nonlinear least-squares problems," IEEE Transactions on Automatic Control, vol. 64, no. 2, pp. 526-537, 2019.

[6] E.-W. Bai, "Identification of linear systems with hard input nonlinearities of known structure," Automatica, vol. 38, no. 5, pp. 853-860, 2002.

[7] D. Wang, L. Li, Y. Ji, and Y. Yan, "Model recovery for Hammerstein systems using the auxiliary model based orthogonal matching pursuit method," Applied Mathematical Modelling, vol. 54, pp. 537-550, 2018.

[8] F. Ding, H. Chen, L. Xu, J. Dai, Q. Li, and T. Hayat, "A hierarchical least squares identification algorithm for Hammerstein nonlinear systems using the key term separation," Journal of the Franklin Institute, vol. 355, no. 8, pp. 37373752, 2018.

[9] D. Wang, Y. Yan, Y. Liu, and J. Ding, "Model recovery for Hammerstein systems using the hierarchical orthogonal matching pursuit method," Journal of Computational and Applied Mathematics, vol. 345, pp. 135-145, 2019.

[10] Z. Ge, F. Ding, L. Xu, A. Alsaedi, and T. Hayat, "Gradientbased iterative identification method for multivariate equation-error autoregressive moving average systems using the decomposition technique," Journal of the Franklin Institute, vol. 356, no. 3, pp. 1658-1676, 2019.

[11] F. Ding, X. Zhang, and L. Xu, "The innovation algorithms for multivariable state-space models," International Journal of Adaptive Control and Signal Processing, vol. 33, no. 11, pp. 1601-1618, 2019.

[12] J. Li and X. Li, "Particle swarm optimization iterative identification algorithm and gradient iterative identification algorithm for Wiener systems with colored noise," Complexity, vol. 2018, Article ID 7353171, 8 pages, 2018.

[13] Y. Gu, J. Liu, X. Li, Y. Chou, and Y. Ji, "State space model identification of multirate processes with time-delay using the expectation maximization," Journal of the Franklin Institute, vol. 356, no. 3, pp. 1623-1639, 2019.

[14] X. Wang and F. Ding, "The filtering based parameter identification for bilinear-in-parameter systems," Journal of the Franklin Institute, vol. 356, no. 1, pp. 514-538, 2019.

[15] X. Zhang, F. Ding, and E. Yang, "State estimation for bilinear systems through minimizing the covariance matrix of the state estimation errors," International Journal of Adaptive Control and Signal Processing, vol. 33, no. 7, pp. 1157-1173, 2019.

[16] Y. Mao, F. Ding, L. Xu, and T. Hayat, "Highly efficient parameter estimation algorithms for Hammerstein non-linear systems," IET Control Theory \& Applications, vol. 13, no. 4, pp. 477-485, 2019.

[17] D. Wang, L. Mao, and F. Ding, "Recasted models-based hierarchical extended stochastic gradient method for MIMO nonlinear systems," IET Control Theory \& Applications, vol. 11, no. 4, pp. 476-485, 2017.

[18] Y. Pu and J. Chen, "A novel maximum likelihood-based stochastic gradient algorithm for Hammerstein nonlinear systems with coloured noise," International Journal of Modelling, Identification and Control, vol. 32, no. 1, pp. 23-29, 2019.

[19] B. Mu, E.-W. Bai, W. X. Zheng, and Q. Zhu, "A globally consistent nonlinear least squares estimator for identification of nonlinear rational systems," Automatica, vol. 77, pp. 322-335, 2017.
[20] Q. Liu and F. Ding, "Auxiliary model-based recursive generalized least squares algorithm for multivariate output-error autoregressive systems using the data filtering," Circuits, Systems, and Signal Processing, vol. 38, no. 2, pp. 590-610, 2019.

[21] Q. M. Zhu, "An implicit least squares algorithm for nonlinear rational model parameter estimation," Applied Mathematical Modelling, vol. 29, no. 7, pp. 673-689, 2005.

[22] D. Wang, Z. Zhang, and B. Xue, "Decoupled parameter estimation methods for Hammerstein systems by using filtering technique," IEEE Access, vol. 6, pp. 66612-66620, 2018.

[23] A. Taha and A. S. Hadi, "A general approach for automating outliers identification in categorical data," in Proceedings of the 2013 ACS International Conference on Computer Systems and Applications (AICCSA), pp. 1-8, Ifrane, Morocco, May 2013.

[24] R. Baragona and F. Battaglia, "Outliers detection in multivariate time series by independent component analysis," Neural Computation, vol. 19, no. 7, pp. 1962-1984, 2007.

[25] Q. Zhu, L. Liu, W. Zhang, and S. Li, "Control of complex nonlinear dynamic rational systems," Complexity, vol. 2018, Article ID 8953035, 12 pages, 2018.

[26] F. Ding, L. Lv, J. Pan et al., “Two-stage gradient-based iterative estimation methods for controlled autoregressive systems using the measurement data," International Journal of Control, Automation and Systems, vol. 18, no. 4, pp. 886-896, 2020.

[27] J. Zhang, Q. Zhu, Y. Li, and X. L. Wu, "Homeomorphism mapping based neural networks for finite time constraint control of a class of nonaffine pure-feedback nonlinear systems," Complexity, vol. 2019, Article ID 9053858, 11 pages, 2019.

[28] Y. J. Zhao, A. Fatehi, and B. Huang, "Robust estimation of ARX models with time-varying time delays using variational Bayesian approach," IEEE Transactions on Cybernetics, vol. 48, no. 2, pp. 532-542, 2017.

[29] X. Jin, B. Huang, and D. S. Shook, "Multiple model LPV approach to nonlinear process identification with EM algorithm," Journal of Process Control, vol. 21, no. 1, pp. 182-193, 2011.

[30] R. A. Horn and C. R. Johnson, Matrix Analysis, Cambridge University Press, Cambridge, UK, 2012.

[31] J. Chen, Q. M. Zhu, J. Li, and Y. J. Liu, "Biased compensation recursive least squares algorithm for rational models," Nonlinear Dynamics, vol. 91, no. 2, pp. 797-807, 2018. 